\title{
Tracking control in billiards using mirrors without smoke, Part II: additional Lyapunov-based local and global results
}

\author{
F. Forni, A.R. Teel, L. Zaccarian
}

\begin{abstract}
Two control results are described: 1) local tracking control for convex billiards with piecewise locally Lipschitz boundary, and 2) global tracking control for special polyhedral billiards, including rectangles and equilateral triangles. The controllers are based on Lyapunov functions and a mirroring concept introduced in a companion paper. The local results require the impacts to satisfy an average dwell-time condition with parameters that depend on the Lipschitz constant of the function that characterizes the boundary. For piecewise constant boundary, and for the global results, the average dwelltime parameters are arbitrary. Tools from stability analysis for hybrid systems are used to establish the results.
\end{abstract}

\section{INTRODUCTION}

This paper is a continuation of [3] where a new Lyapunovbased technique for local tracking in convex, polyhedral billiards is developed. In this paper, we show how the results in [3] can be extended to local tracking in convex billiards with piecewise locally Lipschitz boundary, and to global tracking for some polyhedral billiards. Background material on the billiard tracking problem can be found in [3]. Several techinques addressing tracking on billiards and, in general, on mechanical systems with impacts are provided in the works [1], [4], [6] and references therein.

In Section II, we describe general convex billiards and describe hybrid models for them that capture impacts at the boundaries. In Section III, we describe the control algorithm that achieves local tracking, with tracking results proved in Section IV. In Section V we describe a control algorithm that, under appropriate assumptions, achieves global asymptotic tracking. In Section VI we show that these assumptions hold for certain polyhedral billiards.

\section{SYSTEM DESCRIPTION}

We consider a tracking control problem where we aim to make the variable $x_{p} \in \mathbb{R}^{2}$ track the reference variable $z_{p} \in \mathbb{R}^{2}$ where both variables evolve in a closed, convex set $\mathcal{F} \subset \mathbb{R}^{2}$ that has nonempty interior and a piecewise locally Lipschitz boundary. We refer to the set $\mathcal{F}$ as a billiard. Let $\eta_{\circ}$ denote some point in the interior of $\mathcal{F}$. By piecewise locally Lipschitz boundary, we mean that for each $\eta \in \partial \mathcal{F}$, except possibly for a finite number of points, the conditions

$$
\left\langle F, \eta-\eta_{c}\right\rangle \geq 0 \quad \forall \eta_{c} \in \mathcal{F}, \quad\left\langle F, \eta-\eta_{\circ}\right\rangle=1
$$

Research supported in part by AFOSR grant FA9550-09-1-0203, NSF grants ECCS-0925637 and CNS-0720842, by ENEA-Euratom and MIUR.

F. Forni and L. Zaccarian are with the Dipartimento di Informatica, Sistemi e Produzione, University of Rome Tor Vergata, 00133, Italy, forniedisp.uniroma2.it, zackedisp.uniroma2.it, A.R. Teel is with the ECE Department, University of California, Santa Barbara, CA 93106-9560, USA, teel@ece.ucsb.edu. define a unique vector $F(\eta)$ and any function $F: \partial \mathcal{F} \rightarrow$ $\mathbb{R}^{2} \backslash\{0\}$ satisfying (1) is piecewise locally Lipschitz. Note that 1) $\left\langle F(\eta), \eta-\eta_{c}\right\rangle=0$ implies $\eta_{c} \in \partial \mathcal{F}$, and 2) $\eta_{c} \in \mathcal{F}$ if and only if $\left\langle F(\eta), \eta_{c}-\eta_{\circ}\right\rangle \leq 1$ for each $\eta \in \partial \mathcal{F}$.

Let $x_{v} \in \mathbb{R}^{2}$ and $z_{v} \in \mathbb{R}^{2}$ denote the velocities of $x_{p}$ and $z_{p}$ respectively. Define $x=\left(x_{p}^{T}, x_{v}^{T}\right)^{T}$ and $z=\left(z_{p}^{T}, z_{v}^{T}\right)^{T}$. The variable $z$ is constrained to a compact set $\mathcal{K} \subset \mathcal{F} \times$ $\mathbb{R}^{2}$. The following assumption on the set $\mathcal{K}$ prevents the $z$ state from reaching any billiard "corner" (informally, a point on the boundary of $\mathcal{F}$ where $F$ is discontinuous) and from "sliding" along the boundary of the billiard.

Assumption 1: For the compact set $\mathcal{K}$, there exists $\nu>0$ such that $\left(\eta^{*}, \omega^{*}\right) \in \mathcal{K} \cap\left(\partial \mathcal{F} \times \mathbb{R}^{2}\right)$ implies:

1) $\eta \mapsto F(\eta)$ is locally Lipschitz at $\eta^{*}$,

2) $\left|\left\langle F\left(\eta^{*}\right), \omega^{*}\right\rangle\right| \geq \nu\left|F\left(\eta^{*}\right)\right|$.

During continuous evolution, the equations of motion are

$$
\ddot{z}_{p} \in \alpha(z), \quad \ddot{x}_{p}=\phi(x)+u
$$

where $\phi: \mathcal{F} \times \mathbb{R}^{2} \rightarrow \mathbb{R}^{2}$ is a continuous function, $\alpha: \mathbb{R}^{4} \rightrightarrows$ $\mathbb{R}^{2}$ is a set-valued mapping that is outer semicontinuous and locally bounded having nonempty convex values for each $z \in \mathcal{K}$, and $u \in \mathbb{R}^{2}$ is the control. A special case covered here is when $\alpha$ is replaced by a continuous function defined on $\mathcal{K}$. We allow set-valued accelerations for the reference variable $z_{p}$ in order to allow the possibility that when the reference state returns to a particular value it leaves that value along a trajectory that is different from the trajectory it followed the previous time it was at that value. While the acceleration is not assumed to be unique, the selected acceleration at each time is assumed to be known by the control algorithm.

A variable with position vector $\eta \in \partial \mathcal{F}$ and velocity vector $\omega$ is said to impact the boundary of the billiard if $\langle F(\eta), \omega\rangle \geq$ 0 . That is, impacts occur when $(x, z)$ belong to the set $D_{b}$ defined as $D_{b}:=D_{b 1} \cup D_{b 2}$ where

$$
\begin{aligned}
& D_{b 1}:=\left\{x \in \partial \mathcal{F} \times \mathbb{R}^{2}:\left\langle F\left(x_{p}\right), x_{v}\right\rangle \geq 0\right\} \times \mathcal{K} \\
& D_{b 2}:=\mathcal{F} \times\left\{z \in \mathcal{K} \cap\left(\partial \mathcal{F} \times \mathbb{R}^{2}\right):\left\langle F\left(z_{p}\right), z_{v}\right\rangle \geq 0\right\} .
\end{aligned}
$$

When a variable impacts the billiard boundary at a point where the boundary is locally Lipschitz, the variable's velocity is reflected in a direction that is determined by the velocity and the vector $F$ at the point of impact. These instantaneous changes in velocity can be written as $\omega^{+}=M(F(\eta)) \omega$, where $\omega$ denotes the variable's velocity and

$$
\begin{aligned}
M(F) & :=R(F)^{T} \operatorname{diag}(1,-1) R(F) \\
R(F) & :=\frac{1}{|F|}\left[\begin{array}{c}
F^{T} J \\
F^{T}
\end{array}\right], \quad J=\left[\begin{array}{rr}
0 & -1 \\
1 & 0
\end{array}\right]
\end{aligned}
$$


where $|F|=\sqrt{F^{T} F}$. The positions do not change at impacts, that is, $\eta^{+}=\eta$ where $\eta$ denotes the position of a variable. This rule can be equivalently written as $\eta^{+}=$ $M(F(\eta)) \eta+c(F(\eta))$ where

$$
c(F)=F \cdot 2\left(1+F^{T} \eta_{\circ}\right) /|F|^{2} .
$$

Several useful identities, including $\eta=M(F(\eta)) \eta+c(F(\eta))$ for any $\eta \in \partial \mathcal{F}$, are established in [3]. With the definitions

$$
\begin{aligned}
& \widetilde{M}(F)=\operatorname{diag}(M(F), M(F)) \\
& \widetilde{c}(F)=\left[\begin{array}{cc}
c(F)^{T} & 0_{1 \times 2}
\end{array}\right]^{T} \\
& \widetilde{F}(\eta)=\bigcap_{\delta>0} F((\eta+\delta \mathbb{B}) \cap \partial \mathcal{F}),
\end{aligned}
$$

$\widetilde{F}(\eta)=F(\eta)$ at points where $F$ is Lipschitz and, for all impacts, even at points where the boundary is not smooth,

$$
\left[\begin{array}{l}
\eta \\
\omega
\end{array}\right]^{+} \in \bigcup_{F \in \widetilde{F}(\eta)} \widetilde{M}(F)\left[\begin{array}{l}
\eta \\
\omega
\end{array}\right]+\widetilde{c}(F) .
$$

Also define $F\left(\eta_{\circ}\right)=0, M(0)=I, c(0)=0, \widetilde{M}(0)=I$, $\widetilde{c}(0)=0$, and, for each $(F, z) \in \mathbb{R}^{2} \times \mathcal{F}$, define

$$
m(F, z):=\widetilde{M}(F) z+\widetilde{c}(F)
$$

In order to rule out solutions that always jump and never evolve continuously, we augment the plant with an average dwell-time automaton [2], [5, eq. (S3), (S4)]. Letting $N$ be a positive integer and $\rho>0$, we add the dynamics

$$
\begin{array}{ccc}
\dot{\sigma} & \in[0, \rho] & \sigma \in[0, N] \\
\sigma^{+} & =\sigma-1 & \sigma \in[1, N] .
\end{array}
$$

Jumps of the billiard system are allowed when $\sigma \in[1, N]$ and either $x \in \partial \mathcal{F} \times \mathbb{R}^{2}$ and $\left\langle F\left(x_{p}\right), x_{v}\right\rangle \geq 0$ or $z \in \mathcal{K} \cap$ $\left(\partial \mathcal{F} \times \mathbb{R}^{2}\right)$ and $\left\langle F\left(z_{p}\right), z_{v}\right\rangle \geq 0$. For any hybrid system in which $\sigma$ is inserted, each solution has a hybrid time domain that satisfies $j-i \leq \rho(t-s)+N$ for all $(t, j)$ and $(s, i)$ in the domain that satisfy $t+j \geq s+i$; see [2]. The values $(\rho, N)$ may be set based on the initial value of $x$. Neither $\rho$ nor $N$ will be used in control design but, in the case of billiards with curved boundary, the values of $\rho$ and $N$ will appear in the analysis. The larger the curvature of the billiard walls, the smaller $\rho$ must be to guarantee asymptotic stability using our techniques. Conversely, as the curvature tends toward zero, the allowable value of $\rho$ becomes arbitrarily large.

\section{Control Algorithm}

Our control algorithm is parametrized by a matrix $\bar{P} \in$ $\mathbb{R}^{2 \times 2}$ and real numbers $\bar{k}_{1}, \bar{k}_{2}$ specified as follows.

Assumption 2: $\bar{P}=\bar{P}^{T}>0$ and $\bar{A}_{c l}^{T} \bar{P}+\overline{P A}_{c l} \leq-\lambda \bar{P}$ for some $\lambda>0$ where $\bar{A}_{c l}=\left[\begin{array}{cc}0 & \frac{1}{c l} \\ -\bar{k}_{1} & -\bar{k}_{2}\end{array}\right]$.

Define $P:=\bar{P} \otimes I_{2 \times 2}, K:=\bar{K} \otimes I_{2 \times 2}$ where $\bar{K}:=$ $\left[\begin{array}{cc}\bar{k}_{1} & \bar{k}_{2}\end{array}\right]$, and $|v|_{P}:=\sqrt{v^{T} P v}$ for each $v \in \mathbb{R}^{4}$, and note that for every $T \in \mathbb{R}^{2 \times 2}$ which satisfies $T^{T} T=I$ we have that $|\operatorname{diag}(T, T) v|_{P}=|v|_{P}$. Thus, using $M(F)=M(F)^{T}$, $M(F) M(F)=I$ and $M(F) c(F)=-c(F)$, it follows that

$$
\begin{aligned}
& |x-m(F, z)|_{P}=|m(F, x)-z|_{P} \\
& |m(F(\eta), x)-m(F(\eta), z)|_{P}=|x-z|_{P} .
\end{aligned}
$$

Now augment the dynamics of $(x, z, \sigma)$, which evolves in $\left(\mathcal{F} \times \mathbb{R}^{2}\right) \times \mathcal{K} \times[0, N]$, with the variable $\eta \in \mathbb{R}^{2}$, which evolves in the set $\Pi_{p}(\mathcal{K}) \cap \partial \mathcal{F}$, where

$$
\Pi_{p}(\mathcal{K})=\left\{z_{p} \in \mathcal{F}:\left(z_{p}, z_{v}\right) \in \mathcal{K} \text { for some } z_{v} \in \mathbb{R}^{2}\right\},
$$

i.e., the projection of $\mathcal{K}$ to positions. Define

$$
C:=\left(\mathcal{F} \times \mathbb{R}^{2}\right) \times \mathcal{K} \times[0, N] \times\left(\Pi_{p}(\mathcal{K}) \cap \partial \mathcal{F}\right) .
$$

During continuous-time evolution, which is allowed when $(x, z, \sigma, \eta) \in C$, the variable $\eta$ satisfies $\dot{\eta}=0$. When the $x$ state jumps, which is allowed when $(x, z, \sigma, \eta) \in D_{b 1} \times$ $[1, N] \times\left(\Pi_{p}(\mathcal{K}) \cap \partial \mathcal{F}\right)$ the variable $\eta$ is updated as $\eta^{+}=x_{p}$. When the $z$ state jumps, which is allowed when $(x, z, \sigma, \eta) \in$ $D_{b 2} \times[1, N] \times\left(\Pi_{p}(\mathcal{K}) \cap \partial \mathcal{F}\right)$ the variable $\eta$ is updated as $\eta^{+}=z_{p}$. The control law is

$$
\begin{aligned}
& u=-\phi(x)+M(F(s)) \alpha-K(x-m(F(s), z)) \\
& s \in \operatorname{argmin}_{s \in\left\{\eta, \eta_{\circ}\right\}}|x-m(F(s), z)|_{P}
\end{aligned}
$$

where $\alpha \in \alpha(z)$ represents the acceleration of $z_{p}$ at the current time. Since $\eta \in \Pi_{p}(\mathcal{K}) \cap \partial \mathcal{F}$, it follows from Assumption 1 that, when $z \mapsto \alpha(z)$ is a continuous function, the control law $u$ is a continuous function on the set

$$
\left\{(x, z, \sigma, \eta) \in C:|x-m(0, z)|_{P} \neq|x-m(F(\eta), z)|_{P}\right\} .
$$

The closed-loop tracking system has the flow dynamics (5a), (2), (8), which evolve on the set $C$ in (7), and the jump dynamics given in Section II together with the rules for $\eta^{+}$ specified above (8). Jumps are allowed if the state is in $D:=$ $D_{b} \times[1, N] \times\left(\Pi_{p}(\mathcal{K}) \cap \partial \mathcal{F}\right)$ where $D_{b}$ is defined above (3).

\section{Closed-LOOP RESUlts}

Define

$$
\begin{aligned}
& \mathcal{A}_{\circ}:=\{(x, z, \sigma, \eta) \in C: x=m(0, z)\} \\
& \mathcal{A}_{m}:=\left\{(x, z, \sigma, \eta) \in C: x=m(F(\eta), z), \eta=z_{p}\right\}
\end{aligned}
$$

and $\mathcal{A}:=\mathcal{A}_{\circ} \cup \mathcal{A}_{m}$. The set $\mathcal{A}$ is compact and satisfies

$$
(x, z, \sigma, \eta) \in \mathcal{A} \quad \Longrightarrow \quad x_{p}=z_{p} .
$$

We establish that $\mathcal{A}$ is locally asymptotically stable (LAS) under appropriate assumptions on $(\rho, N)$. LAS of the set $\mathcal{A}$ for the closed-loop system with state $X=(x, z, \sigma, \eta)$ entails 1) (stability) for each $\varepsilon>0$ there exists $\delta>0$ such that $|X(0,0)|_{\mathcal{A}} \leq \delta \quad \Longrightarrow \quad|X(t, j)|_{\mathcal{A}} \leq \varepsilon \quad \forall(t, j) \in \operatorname{dom} X$, where $|X|_{\mathcal{A}}$ is the distance of the point $X$ to the set $\mathcal{A}$, and 2) (attractivity) there exists $\mu>0$ such that for $|X(0,0)|_{\mathcal{A}} \leq$ $\mu$, each solution is bounded and each complete solution converges to $\mathcal{A}$, that is, satisfies $\lim _{t+j \rightarrow \infty}|X(t, j)|_{\mathcal{A}}=0$.

Using the next definition, we can state the main result of the section. A function $\varrho: \mathbb{R}_{>0} \rightarrow \mathbb{R}_{>0}$ belongs to class $\widehat{\mathcal{L}}$ if it is continuous, nonincreasing, $\lim _{s \rightarrow \infty} \varrho(s)=0$ and $\lim _{s \rightarrow 0} \varrho(s)=\infty$.

Theorem 1: Let Assumptions 1 and 2 hold and let the positive integer $N$ be given. There exists $\varrho \in \widehat{\mathcal{L}}$ such that, if $\eta \mapsto F(\eta)$ is Lipschitz of rank $L>0$ for all $\eta \in \Pi_{p}(\mathcal{K}) \cap \partial \mathcal{F}$, then for each $\rho \leq \varrho(L)$, the compact set $\mathcal{A}$ is locally asymptotically stable for the closed-loop tracking system.

The next corollary, which follows from Theorem 1 and (9), establishes a local tracking result for the closed-loop system. 
Corollary 1: Under the conditions of Theorem 1, the solutions of the closed-loop tracking system satisfy:

1) for each $\varepsilon>0$ there exists $\delta>0$ such that $|X(0,0)|_{\mathcal{A}} \leq \delta$ implies $\left|x_{p}(t, j)-z_{p}(t, j)\right| \leq \varepsilon$ for all $(t, j) \in \operatorname{dom} X$;

2) there exists $\mu>0$ such that, for $|X(0,0)|_{\mathcal{A}} \leq \mu$, each complete solution, $\lim _{t+j \rightarrow \infty}\left|x_{p}(t, j)-z_{p}(t, j)\right|=0$.

Proof of Theorem 1:

Given $\kappa>0$, we define

$$
\begin{aligned}
\mathcal{R}_{\kappa}:=\{(x, z, \sigma, \eta) \in C: & \\
& \left.\max \left\{\left|x_{p}-\eta\right|,\left|z_{p}-\eta\right|\right\} \leq \kappa|x-m(F(\eta), z)|\right\} .
\end{aligned}
$$

The proof is based on the next lemma.

Lemma 1: There exist $\kappa>0$ and a compact set $\Omega$ contained in $\left(\mathcal{A}_{\circ}+\varepsilon \mathbb{B}\right) \cup\left(\left(\mathcal{A}_{m}+\varepsilon \mathbb{B}\right) \cap \mathcal{R}_{\kappa}\right)$ that is globally asymptotically stable for the closed-loop tracking system when the flow and jump sets are intersected with $\mathcal{A}+\varepsilon \mathbb{B}$ with $\varepsilon>0$ sufficiently small.

Thus, we restrict the flow and jump sets to $\mathcal{A}+\varepsilon \mathbb{B}$ for some $\varepsilon>0$ sufficiently small. According to [5, Corollary 19], is it enough to establish global asymptotic stability for the closedloop system with the flow and jump sets further restricted to $\Omega$. We consider the evolution of the Lyapunov function candidate

$$
V(x, z, \eta)=\min _{s \in\left\{\eta, \eta_{\circ}\right\}}|x-m(F(s), z)|_{P}^{2} .
$$

With the restriction that the state belongs to $\Omega$, it follows that $V(x, z, \eta)=0$ if and only if $(x, z, \eta) \in \mathcal{A}$.

Due to the choice for the control in (8), it follows that during continuous-time evolution we have

$$
\dot{V}(x, z, \eta) \leq-\lambda \min _{s \in\left\{\eta, \eta_{\circ}\right\}}|x-m(F(s), z)|_{P}^{2} .
$$

For each $\eta \in \Pi_{p}(\mathcal{K}) \cap \partial \mathcal{F}$, we define $e(\eta, x, z)=$ $m(F(\eta), z)-m\left(F\left(x_{p}\right), z\right)$ and we define $e\left(\eta_{\circ}, x, z\right)=0$. For jumps in the plant where the state $x$ impacts the boundary of the billiard, we have

$$
\begin{aligned}
V\left(x^{+}, z^{+}, \eta^{+}\right) & =\min _{s \in\left\{\eta^{+}, \eta_{\circ}\right\}}\left|x^{+}-m(F(s), z)\right|_{P}^{2} \\
& =\min _{s \in\left\{x_{p}, \eta_{\circ}\right\}}\left|m\left(F\left(x_{p}\right), x\right)-m(F(s), z)\right|_{P}^{2} \\
& =\min _{P}\left\{\left|m\left(F\left(x_{p}\right), x\right)-z\right|_{P}^{2},|x-z|_{P}^{2}\right\} \\
& =\min _{s \in\left\{\eta_{\circ}, x_{p}\right\}}|x-m(F(s), z)|_{P}^{2} \\
& =\min _{s \in\left\{\eta, \eta_{\circ}\right\}}|x-m(F(s), z)+e(s, x, z)|_{P}^{2} .
\end{aligned}
$$

where we used (6) from the second to the fourth line. When $z$ jumps, using again (6), we have

$$
\begin{aligned}
V\left(x^{+}, z^{+}, \eta^{+}\right) & =\min _{s \in\left\{\eta^{+}, \eta_{\circ}\right\}}\left|x-m\left(F(s), z^{+}\right)\right|_{P}^{2} \\
& =\min _{s \in\left\{z_{p}, \eta_{\circ}\right\}}\left|x-m\left(F(s), m\left(F\left(z_{p}\right), z\right)\right)\right|_{P}^{2} \\
& =\min _{P}\left\{|x-z|_{P}^{2},\left|x-m\left(F\left(z_{p}\right), z\right)\right|_{P}^{2},\right\} \\
& =\min _{s \in\left\{\eta_{\circ}, z_{p}\right\}}|m(F(s), x)-z|_{P}^{2} \\
& =\min _{s \in\left\{\eta, \eta_{\circ}\right\}}|m(F(s), x)-z+e(s, z, x)|_{P}^{2}
\end{aligned}
$$

Using that $\mathcal{K}$ is compact, $F(\cdot)$ is Lipschitz with Lipschitz constant $L$ near $\Pi_{p}(\mathcal{K}) \cap \partial \mathcal{F}$, and the definitions of $m(\cdot, z)$ in (4), $e$ above and $\mathcal{R}_{\kappa}$ in (10), there exists $\widetilde{\kappa}>0$ such that, if $|x-m(F(\eta), z)|_{P}^{2} \leq|x-z|_{P}^{2}$ then

$$
\max \{|e(\eta, x, z)|,|e(\eta, z, x)|\} \leq \widetilde{\kappa} L|x-m(F(\eta), z)| .
$$

Using that $\left(v_{1}+v_{2}\right)^{T} P\left(v_{1}+v_{2}\right)=v_{1}^{T} P v_{1}+2 v_{1}^{T} P v_{2}+$ $v_{2}^{T} P v_{2} \leq v_{1}^{T} P v_{1}+\lambda_{\max }(P)\left|v_{2}\right|\left(2\left|v_{1}\right|+\left|v_{2}\right|\right)$, it follows that there exists $\widehat{\kappa}>0$ such that

$$
V\left(x^{+}, z^{+}, \eta^{+}\right) \leq(1+\widehat{\kappa} L(1+L)) V(x, z, \eta) .
$$

Now we define $Y(x, z, \sigma, \eta):=\exp (\lambda \sigma / 2 \rho) V(x, z, \eta)$ and we ask that $\exp (-\lambda / 2 \rho)(1+\widehat{\kappa} L(1+L)):=\lambda_{d}<1$. In fact, we can fix $\lambda_{d}<1$ and then solve for $\rho$ to get a function $\varrho \in \widehat{\mathcal{L}}$. Then $\dot{Y}(x, z, \sigma, \eta) \leq-\frac{\lambda}{2} Y(x, z, \sigma, \eta)$ and

$$
\begin{aligned}
Y\left(x^{+}, z^{+}\right. & \left., \sigma^{+}, \eta^{+}\right) \\
& \leq \exp \left(-\frac{\lambda}{2 \rho}\right)(1+\widehat{\kappa} L(1+L)) Y(x, z, \sigma, \eta) \\
& =\lambda_{d} Y(x, z, \sigma, \eta) .
\end{aligned}
$$

This establishes the desired result.

\section{Global RESUlts FOR SPECial BILliardS}

\section{A. Assumptions}

Henceforth, we pursue global asymptotic tracking. That is, we seek a control algorithm causing $x_{p}$ to asymptotically track $z_{p}$ from any initial condition $(x, z) \in\left(\mathcal{F} \times \mathbb{R}^{2}\right) \times \mathcal{K}$. We limit our attention to special polyhedral billiards, in which case the boundary of the billiard is characterized by a finite set of vectors $F_{i}, i \in\{1, \ldots, k\}$ where $k$ is the number of walls of the billiard, that satisfy (1) (see [3] for details).

We drop the "no sliding" condition in Assumption 1, instead relying on the average dwell-time mechanism of the model (see (5)) to prevent an infinite number of jumps on a boundary. Moreover, at this point we do not need to make the "no corners" condition of Assumption 1 explicit, as it is covered implicitly by the following assumption on the existence of a family of control Lyapunov functions. Later we justify this assumption for special billiards by assuming that the $z$ variable does not reach the corners of the billiard.

Assumption 3: The matrix $P=P^{T} \in \mathbb{R}^{4 \times 4}$, the matrix $K \in \mathbb{R}^{2 \times 4}$, and the $Q+1(Q \geq k)$ affine functions $z \mapsto n_{i}(z)$ satisfy the following properties:

1) $n_{i}(z)=m\left(F_{i}, z\right)$ for $i \in\{0, \ldots, k\}$;

2) for each $i \in\{k+1, \ldots, Q\}$, there exist $T_{i} \in \mathbb{R}^{2 \times 2}$ and $L_{i} \in \mathbb{R}^{2}$ such that $n_{i}(z)=\widetilde{T}_{i} z+\widetilde{L}_{i}$ where

$$
\widetilde{T}_{i}:=\left[\begin{array}{cc}
T_{i} & 0 \\
0 & T_{i}
\end{array}\right], \quad \widetilde{L}_{i}:=\left[\begin{array}{c}
L_{i} \\
0
\end{array}\right] ;
$$

3) Defining $A_{c l}=\left[\frac{0_{2 \times 2} I_{2 \times 2}}{K}\right]$, $A_{c l}^{T} P+P A_{c l} \leq-H^{T} H$ where $\left(H, A_{c l}\right)$ is observable;

4) For each jump of $(x, z)$,

$$
\min _{i \in\{0, \ldots, Q\}}\left|x^{+}-n_{i}\left(z^{+}\right)\right|_{P}^{2} \leq \min _{i \in\{0, \ldots, Q\}}\left|x-n_{i}(z)\right|_{P}^{2} ;
$$

5) For each $(x, z) \in\left(\mathcal{F} \times \mathbb{R}^{2}\right) \times \mathcal{K}$,

$$
\min _{i \in\{0, \ldots, Q\}}\left|x-n_{i}(z)\right|=0 \Longrightarrow \min _{i \in\{0, \ldots, k\}}\left|x-n_{i}(z)\right|=0 .
$$

We typically establish the last condition by eliminating corners from $\mathcal{K}$ and then showing that if $z_{p}$ is not at a corner then $x \neq n_{i}(z)$ for $i \in\{k+1, \ldots, Q\}$. 


\section{B. Control algorithm}

Paralleling the state $\eta$ of Section III, our controller uses an index variable $q \in \mathbb{R}$, whose values are restricted to the finite set $\mathcal{Q}:=\{0, \ldots, Q\}$. During continuous-time evolution, which is allowed when

$$
(x, z, \sigma, q) \in C:=\left(\mathcal{F} \times \mathbb{R}^{2}\right) \times \mathcal{K} \times[0, N] \times \mathcal{Q},
$$

we use $\dot{q}=0$. At jumps, which are allowed when

$$
(x, z, \sigma, q) \in D:=D_{b} \times[1, N] \times \mathcal{Q},
$$

(where $D_{b}$ is defined above (3)) $q$ is reset to any value $q^{+} \in$ $\mathcal{Q}$ satisfying

$$
\left|x^{+}-n_{q^{+}}\left(z^{+}\right)\right|_{P}^{2}=\min _{i \in \mathcal{Q}}\left|x^{+}-n_{i}\left(z^{+}\right)\right|_{P}^{2} .
$$

The control law is selected, with the knowledge of the current acceleration of the $z$-ball, in order to satisfy

$$
\left[\begin{array}{c}
\ddot{z}_{p} \\
\ddot{x}_{p}
\end{array}\right] \in \widehat{\alpha}(x, z, q)
$$

where $\widehat{\alpha}(x, z, q)=\left\{\left(a_{1}, a_{2}\right) \in \mathbb{R}^{2} \times \mathbb{R}^{2}: a_{1} \in \alpha(z), a_{2}=\right.$ $\left.T_{q} a_{1}+K\left(x-n_{q}(z)\right)\right\}$ In particular, with $\widetilde{x}=x-n_{q}(z)$, we have $\dot{\tilde{x}}=A_{c l} \widetilde{x}$. Together with the dynamics in Section II, this defines the hybrid closed-loop tracking system. Define

$$
\mathcal{A}:=\left\{(x, z, \sigma, q) \in C: x=n_{q}(z)\right\}
$$

and note that if $(x, z, \sigma, q) \in \mathcal{A}$ then $\min _{i \in \mathcal{Q}}\left|x-n_{i}(z)\right|=$ 0 which implies, by assumption, that $\min _{i \in\{1, \ldots, k\}} \mid x-$ $n_{i}(z) \mid=0$. This, in turn, implies that $x_{p}=z_{p}$. Thus, like for the local results, asymptotic stability of the set $\mathcal{A}$ implies asymptotic tracking. We have the following result.

Theorem 2: Under Assumption 3, for each $\rho>0$ and $N>0$, the compact set $\mathcal{A}$ is globally exponentially stable for the closed-loop system tracking system.

Remark 1: For given $(\rho, N)$, the dwell-time automaton (5) may terminate prematurely solutions which produce a large number of impacts before settling into tracking. Premature termination can be addressed by selecting $\rho$ and $N$ sufficiently large, based on the size of the initial value of $x$.

Proof sketch. We analyze the behavior of the system by starting with the Lyapunov function $W(x, z, q):=\mid x-$ $\left.n_{q}(z)\right|_{P} ^{2}$. By definition of $\mathcal{A}$ in (17), $W$ is positive definite with respect to $\mathcal{A}$. Also $W(x, z, q)$ grows unbounded as $x$ grows unbounded.

Using Assumption 3, during continuous-time evolution

$$
\dot{W}(x, z, q) \leq-\left|x-n_{q}(z)\right|_{H^{T} H}^{2}=-|\widetilde{x}|_{H^{T} H}^{2},
$$

and at jumps

$$
\begin{aligned}
W\left(x^{+}, z^{+}, q^{+}\right) & =\left|x^{+}-n_{q^{+}}\left(z^{+}\right)\right|_{P}^{2} \\
& =\min _{i \in \mathcal{Q}}\left|x^{+}-n_{i}\left(z^{+}\right)\right|_{P}^{2} \\
& \leq \min _{i \in \mathcal{Q}}\left|x-n_{i}(z)\right|_{P}^{2} \\
& \leq\left|x-n_{q}(z)\right|_{P}^{2}=W(x, z, q) .
\end{aligned}
$$

Global asymptotic stability follows from observability of $\left(H, A_{c l}\right)$, the average dwell-time constraint imposed by (5), and the invariance principle [7]. Global exponential stability can be established by using [8, Theorem 2].

\section{SUFFICIENT CONDITIONS FOR ASSUMPTION 3}

\section{A. Preliminaries}

Henceforth, we impose the following assumptions.

Assumption 4: For each $\eta^{*} \in \Pi_{p}(\mathcal{K}) \cap \partial \mathcal{F}$, we have that $\eta \mapsto F(\eta)$ is constant near $\eta^{*}$.

Note that Assumption 4 holds for free in the case of singlewalled and parallel-walled billiards.

Assumption 5: The matrices $K$ and $P=P^{T}>0$ have the form $K:=\bar{K} \otimes I_{2 \times 2}$, where $\bar{K}:=\left[\begin{array}{cc}\bar{k}_{1} & \bar{k}_{2}\end{array}\right]$, and $P=\bar{P} \otimes I_{2 \times 2}$ where the matrix $\bar{P}$ is positive definite and, with the definition $\bar{A}_{c l}=\left[\begin{array}{cc}0 & 1 \\ -\bar{k}_{1} & -\bar{k}_{2}\end{array}\right]$ satisfies $\bar{A}_{c l}^{T} \bar{P}+\overline{P A}_{c l} \leq$ $-\bar{H}^{T} \bar{H}$ where $\left(\bar{H}, \bar{A}_{c l}\right)$ is observable.

One possibility is to take

$$
\bar{P}=\left[\begin{array}{cc}
\bar{k}_{1} & 0 \\
0 & 1
\end{array}\right]
$$

which is used when two parallel walls comprise the billiard.

As observed previously, if $T^{T} T=I$ then

$$
\begin{aligned}
\mid x & -\operatorname{diag}(T, T) z-\left.L\right|_{P} ^{2} \\
& =\left|\operatorname{diag}\left(T^{T}, T^{T}\right) x-z-\operatorname{diag}\left(T^{T}, T^{T}\right) L\right|_{P}^{2} \\
& =\left|z-\operatorname{diag}\left(T^{T}, T^{T}\right) x+\operatorname{diag}\left(T^{T}, T^{T}\right) L\right|_{P}^{2} .
\end{aligned}
$$

So if we have a family of affine functions $z \mapsto n_{i}(z)$ that includes $\operatorname{diag}(T, T) z+L$ and $\operatorname{diag}\left(T^{T}, T^{T}\right) z-\operatorname{diag}\left(T^{T}, T^{T}\right) L$ where $T^{T} T=T T^{T}=I$ then we need to check jump behavior for $z$ only, since the required jump behavior for $x$ will follow by symmetry.

\section{B. A single wall}

Proposition 1: Let $k=1$. Under Assumption 5, Assumption 3 holds with $Q=1$.

Proof. Let $F_{0}=0$ and $F_{1}=F$. Since $M\left(F_{i}\right) c\left(F_{i}\right)=-c\left(F_{i}\right)$ for $i \in\{0,1\}$, according to the observation at the end of the last subsection, we just need to establish that

$$
\left.\min _{i \in\{0,1\}} \mid x-m\left(F_{i}, m(F, z)\right)\right)\left.\right|_{P} ^{2}=\min _{i \in\{0,1\}}\left|x-m\left(F_{i}, z\right)\right|_{P}^{2} .
$$

This relation follows from the fact that $m(0, m(F, z))=$ $m\left(F_{1}, z\right)$ and $m(F, m(F, z))=z$.

\section{Two parallel walls}

Proposition 2: Let $k=2, F_{1} /\left|F_{1}\right|=-F_{2} /\left|F_{2}\right|$, and $\bar{P}$ have the form given in (20). Under Assumption 5, Assumption 3 holds with $Q=2$.

Proof. Note that if $F_{2}^{T}\left(z_{p}-\eta_{\circ}\right)=1$ then $F_{1}^{T}\left(z_{p}-\eta_{\circ}\right) /\left|F_{1}\right|=$ $-1 /\left|F_{2}\right|$. Then, using that $F_{1}^{T} x_{p} \leq 1+F_{1}^{T} \eta_{\circ}$, we have that if $F_{2}^{T}\left(z_{p}-\eta_{\circ}\right)=1$ then

$$
\begin{aligned}
\mid x_{p} & -z_{p}-\left.2 \frac{F_{1}}{\left|F_{1}\right|}\left(\frac{\left|F_{1}\right|+\left|F_{2}\right|}{\left|F_{1}\right|\left|F_{2}\right|}\right)\right|^{2}-\left|x_{p}-z_{p}\right|^{2} \\
& =4\left(\frac{\left|F_{1}\right|+\left|F_{2}\right|}{\left|F_{1}\right|\left|F_{2}\right|}\right)^{2}-4 \frac{F_{1}^{T}}{\left|F_{1}\right|}\left(x_{p}-z_{p}\right)\left(\frac{\left|F_{1}\right|+\left|F_{2}\right|}{\left|F_{2}\right|\left|F_{1}\right|}\right) \\
& \geq 4\left(\left(\frac{\left|F_{1}\right|+\left|F_{2}\right|}{\left|F_{1}\right|\left|F_{2}\right|}\right)^{2}-\left(\frac{1}{\left|F_{1}\right|}+\frac{1}{\left|F_{2}\right|}\right)\left(\frac{\left|F_{1}\right|+\left|F_{2}\right|}{\left|F_{2}\right|\left|F_{1}\right|}\right)\right)=0
\end{aligned}
$$

Note also that $M\left(F_{1}\right)=M\left(F_{2}\right), M\left(F_{1}\right) M\left(F_{2}\right)=$ $M\left(F_{2}\right) M\left(F_{1}\right)=I$ and $c\left(F_{1}\right)-c\left(F_{2}\right)=$ 


$$
\begin{aligned}
2 F_{1} /\left|F_{1}\right|\left(\left.\left|F_{1}\right|^{-1}|+| F_{2}\right|^{-1}\right) \text { so that } \\
\begin{aligned}
m\left(F_{1}, m\left(F_{2}, z\right)\right) & =z+\widetilde{M}\left(F_{1}\right) \widetilde{c}\left(F_{2}\right)+\widetilde{c}\left(F_{1}\right) \\
& =z-\widetilde{c}\left(F_{2}\right)+\widetilde{c}\left(F_{1}\right) \\
& =z+2\left[\begin{array}{c}
\frac{F_{1}}{\left|F_{1}\right|} \\
0_{2 \times 1}
\end{array}\right]\left(\frac{\left|F_{1}\right|+\left|F_{2}\right|}{\left|F_{2}\right|\left|F_{1}\right|}\right) .
\end{aligned}
\end{aligned}
$$

In turn, it follows from the structure of $\bar{P}$ that if $F_{2}^{T}\left(z_{p}-\right.$ $\left.\eta_{\circ}\right)=1$ then $\left|x-m\left(F_{1}, m\left(F_{2}, z\right)\right)\right|_{P}^{2} \geq|x-z|_{P}^{2}$ and also $\left|x-m\left(F_{1}, z\right)\right|_{P}^{2} \geq\left|x-m\left(F_{2}, z\right)\right|_{P}^{2}$. We then have, for jumps of $z$ on the second wall, that

$$
\begin{aligned}
& \min _{i \in\{0,1,2\}}\left|x-m\left(F_{i}, m\left(F_{2}, z\right)\right)\right|_{P}^{2} \\
= & \min \left\{\left|x-m\left(F_{2}, z\right)\right|_{P}^{2},\left|x-m\left(F_{1}, m\left(F_{2}, z\right)\right)\right|_{P}^{2},|x-z|_{P}^{2}\right\} \\
= & \min \left\{\left|x-m\left(F_{2}, z\right)\right|_{P}^{2},|x-z|_{P}^{2}\right\} \\
= & \min \left\{\left|x-m\left(F_{2}, z\right)\right|_{P}^{2},\left|x-m\left(F_{1}, z\right)\right|_{P}^{2},|x-z|_{P}^{2}\right\} .
\end{aligned}
$$

This calculation establishes the result.

\section{Two orthogonal walls}

Proposition 3: Let $k=2$ and $F_{1}^{T} F_{2}=0$. Under Assumptions 4 and 5, Assumption 3 holds with $Q=3$.

Proof. Let $n_{3}(z):=m\left(F_{1}, m\left(F_{2}, z\right)\right)=m\left(F_{2}, m\left(F_{1}, z\right)\right)$. Since $\left.n_{3}\left(m\left(F_{2}, z\right)\right)=m\left(F_{1}, z\right)\right)$, it follows that

$$
\min _{i \in\{0,1,2,3\}}\left|x-n_{i}\left(m\left(F_{2}, z\right)\right)\right|_{P}^{2}=\min _{i \in\{0,1,2,3\}}\left|x-n_{i}(z)\right|_{P}^{2} .
$$

By symmetry

$$
\min _{i \in\{0,1,2,3\}}\left|x-n_{i}\left(m\left(F_{1}, z\right)\right)\right|_{P}^{2}=\min _{i \in\{0,1,2,3\}}\left|x-n_{i}(z)\right|_{P}^{2} .
$$

Finally, to establish the last condition of Assumption 3, it is sufficient to establish that

$$
z_{p} \in \Pi_{p}(\mathcal{K}) \Longrightarrow M\left(F_{2}\right)\left(M\left(F_{1}\right) z_{p}+c\left(F_{1}\right)\right)+c\left(F_{2}\right) \notin \mathcal{F} .
$$

Intuitively, the map $z_{p} \mapsto M\left(F_{1}\right)\left(M\left(F_{2}\right) z_{p}+c\left(F_{2}\right)\right)+c\left(F_{1}\right)$ is a rotation of 180 degrees around the corner point where the walls meet. The only point in the billiard that is not carried out of the billiard by this operation is the corner point, which is excluded by Assumption 4. Rigorously, for $j \in\{1,2\}$,

$$
F_{j}^{T}\left[M\left(F_{2}\right)\left(M\left(F_{1}\right) z_{p}+c\left(F_{1}\right)\right)+c\left(F_{2}\right)\right] \leq 1+F_{j}^{T} \eta_{\circ}
$$

implies $F_{j}^{T} z_{p}=1+F_{j}^{T} \eta_{\mathrm{o}}$. In other words, the condition $M\left(F_{2}\right)\left(M\left(F_{1}\right) z_{p}+c\left(F_{1}\right)\right)+c\left(F_{2}\right) \in \mathcal{F}$ implies that $z_{p}$ is located at the corner of the walls, meaning $z_{p} \notin \Pi_{p}(\mathcal{K})$.

\section{E. Rectangles}

Proposition 4: Let $k=4, F_{1}^{T} F_{2}=0, F_{1}^{T} F_{4}=0$, $F_{1} /\left|F_{1}\right|=-F_{3} /\left|F_{3}\right|$ and $\bar{P}$ have the form given in (20). Under Assumptions 4 and 5, Assumption 3 holds with $Q=8$. Proof. Using the results of the previous subsection, one extra function is constructed for each pair of orthogonal walls. The new situation that must be accounted for is the change in one of these extra "corner" functions when there is an impact on one of the two other walls that do not form the given corner. Let the corner be associated with walls 1 and 2 so that the affine corner function is given as $z \mapsto m\left(F_{1}, m\left(F_{2}, z\right)\right)$. Let wall 3 be parallel to wall 1 , and thus orthogonal to wall 2 .
Then, using the calculations from the cases of parallel and orthogonal walls,

$$
\begin{aligned}
m\left(F_{1}, m\right. & \left.\left(F_{2}, m\left(F_{3}, z\right)\right)\right) \\
& =m\left(F_{1}, m\left(F_{3}, m\left(F_{2}, z\right)\right)\right) \\
& =m\left(F_{2}, z\right)+2\left[\begin{array}{c}
\frac{F_{1}}{\left|F_{1}\right|} \\
0_{2 \times 1}
\end{array}\right]\left(\frac{\left|F_{1}\right|+\left|F_{3}\right|}{\left|F_{3}\right|\left|F_{1}\right|}\right) .
\end{aligned}
$$

Due to the fact that walls 2 and 3 are orthogonal, it follows that if $F_{3}^{T}\left(z_{p}-\eta_{\circ}\right)=1$ then $F_{3}^{T}\left(M\left(F_{2}\right) z_{p}+c\left(F_{2}\right)-\eta_{\circ}\right)=1$. Therefore, following the calculations in the case of parallel walls and using that $\bar{P}$ has the form given in (20), we get that if $F_{3}^{T}\left(z_{p}-\eta_{\circ}\right)=1$ then $\left|x+m\left(F_{1}, m\left(F_{2}, m\left(F_{3}, z\right)\right)\right)\right|_{P}^{2} \geq$ $\left|x-m\left(F_{2}, z\right)\right|_{P}^{2}$ and also $\left|x+m\left(F_{1}, m\left(F_{2}, z\right)\right)\right|_{P}^{2} \geq \mid x-$ $\left.m\left(F_{2}, m\left(F_{3}, z\right)\right)\right|_{P} ^{2}$. The rest of the proof follows the proof for the case of parallel walls.

\section{F. Two walls that meet at special acute angles}

Proposition 5: Let $k=2$, and $F_{1}^{T} F_{2}=\cos (\theta)\left|F_{1}\right|\left|F_{2}\right|$ where $\theta \in\left(0, \frac{\pi}{3}\right]$ satisfies $\ell \theta=\pi$ for some integer $\ell \geq 3$. Under Assumptions 4 and 5, Assumption 3 holds with $Q=$ $2 \ell-1$.

Proof. To save on notation, we use $M_{i}:=M\left(F_{i}\right)$ and $c_{i}:=$ $c\left(F_{i}\right)$. We order the walls such that

$$
M_{1} M_{2}=\left(M_{2} M_{1}\right)^{T}=\left[\begin{array}{rr}
\cos (2 \theta) & \sin (2 \theta) \\
-\sin (2 \theta) & \cos (2 \theta)
\end{array}\right] .
$$

Suppose $\ell$ is even so that $\left(M_{1} M_{2}\right)^{\ell / 2}=\left(M_{2} M_{1}\right)^{\ell / 2}=-I$. Define $T_{-1}=T_{0}=I$. For $i \in\left\{1, \ldots, \frac{\ell}{2}\right\}$, define

$$
\begin{aligned}
T_{4(i-1)+1}:=\left(M_{1} M_{2}\right)^{i-1} M_{1} \\
T_{4(i-1)+2}:=\left(M_{2} M_{1}\right)^{i-1} M_{2} \\
T_{4(i-1)+3}:=\left(M_{2} M_{1}\right)^{i} \\
T_{4(i-1)+4}:=\left(M_{1} M_{2}\right)^{i} \\
L_{4(i-1)+1}:=\sum_{j=0}^{i-1}\left(M_{1} M_{2}\right)^{j} c_{1}+\sum_{j=0}^{i-2}\left(M_{1} M_{2}\right)^{j} M_{1} c_{2} \\
L_{4(i-1)+2}:=\sum_{j=0}^{i-1}\left(M_{2} M_{1}\right)^{j} c_{2}+\sum_{j=0}^{i-2}\left(M_{2} M_{1}\right)^{j} M_{2} c_{1} \\
L_{4(i-1)+3}:=\sum_{j=0}^{i-1}\left(M_{2} M_{1}\right)^{j} c_{2}+\sum_{j=0}^{i-1}\left(M_{2} M_{1}\right)^{j} M_{2} c_{1} \\
L_{4(i-1)+4}:=\sum_{j=0}^{i-1}\left(M_{1} M_{2}\right)^{j} c_{1}+\sum_{j=0}\left(M_{1} M_{2}\right)^{j} M_{1} c_{2} .
\end{aligned}
$$

Note that $T_{2 \ell}=T_{2 \ell-1}=-I, L_{2 \ell}=L_{2 \ell-1}$. Also, $T_{4(i-1)+1}=T_{4(i-1)+1}^{T}, T_{4(i-1)+2}=T_{4(i-1)+2}^{T}, T_{4(i-1)+3}=$ $T_{4(i-1)+4}^{T}$, and

$$
\begin{aligned}
& T_{4(i-1)+1} L_{4(i-1)+1}=-L_{4(i-1)+1} \\
& T_{4(i-1)+2} L_{4(i-1)+2}=-L_{4(i-1)+2} \\
& T_{4(i-1)+3} L_{4(i-1)+4}=-L_{4(i-1)+3} \\
& T_{4(i-1)+4} L_{4(i-1)+3}=-L_{4(i-1)+4} .
\end{aligned}
$$

Furthermore, it can be verified that, for each $i \in$ $\{1, \ldots, 2 \ell-2\}$ and each $j \in\{1,2\}$,

$$
\begin{aligned}
T_{i} M_{j} & =T_{i+(-1)^{i+j-1}(2(j-1)+1)} \\
T_{2 \ell-1} M_{j} & =T_{2 \ell-1-j} \\
T_{i} c_{j}+L_{i} & =L_{i+(-1)^{i+j-1}(2(j-1)+1)} .
\end{aligned}
$$


Finally, we must establish that, for each $j \in$ $\{3, \ldots, 2 \ell-1\}$, each map $z \mapsto T_{j} z+L_{j}$ takes points in $\mathcal{F}$ to points outside of $\mathcal{F}$, except at the corner point. For $j=4(i-1)+3$ and $j=4(i-1)+4, i \in\{1, \ldots, \ell / 2\}$ the mapping is a rotation of $2 i \theta$ radians around the corner point. Since $\ell \theta=\pi$, we have that $2 i \theta \in[2 \theta, \pi]$. For $j=4(i-1)+1$ and $j=4(i-1)+2, i \in\{2, \ldots, \ell / 2\}$, the mapping is a mirroring reflection about a wall, followed by a rotation of $2(i-1) \theta$ radians. The mirroring operation is not a pointwise rotation, but its action is to rotate the domain of the billiard to a set that is a rotation of the billiard by $\theta$ radians. The net effect is a rotation of the billiard by $(2 i-1) \theta$ radians. We have $(2 i-1) \theta \in[3 \theta, \pi-\theta]$. This establishes the result.

The analysis for $\ell$ odd is similar. In this case $\left(M_{1} M_{2}\right)^{(\ell-1) / 2} M_{1}=\left(M_{2} M_{1}\right)^{(\ell-1) / 2} M_{2}$ and the last two pairs $\left(T_{i}, L_{i}\right)$ of the construction for $\ell$ even are dropped.

\section{G. Equilateral triangles}

With results of previous subsections, we get the following.

Proposition 6: Let $k=3$ and let $\bar{P}$ have the form in (20) and let $F_{i}, i \in\{1,2,3\}$ be such that the billiard is an equilateral triangle. Under Assumptions 4 and 5, Assumption 3 holds with $Q=12$.

Proof. Using Proposition 5, we add 3 functions at each corner. So, there is one function for each wall, plus three for each corner, making for 12 extra functions. To verify (3), we investigate what happens to the functions associated with the corner of walls 1 and 2 due to impacts on wall 3 . It can be verified that

$$
\begin{aligned}
& m\left(F_{1}, m\left(F_{2}, m\left(F_{1}, z\right)\right)\right) \\
& =m\left(F_{3}, z\right)+\widetilde{c}\left(F_{1}\right)-\widetilde{c}\left(F_{3}\right)+\widetilde{M}\left(F_{1}\right) \widetilde{M}\left(F_{2}\right)\left(\widetilde{c}\left(F_{1}\right)-\widetilde{c}\left(F_{2}\right)\right) \\
& =m\left(F_{3}, z\right)+\widetilde{v}
\end{aligned}
$$

where $v$ has the form $\widetilde{v}=\left[\begin{array}{ll}v^{T} & 0\end{array}\right]^{T}$ where $F_{3}^{T} v=$ $|v|\left|F_{3}\right|$, i.e., $v$ points in the same direction as $F_{3}$, and the magnitude of $v$ is equal to twice the height of the triangle. In turn, $m\left(F_{1}, m\left(F_{2}, m\left(F_{1}, m\left(F_{3}, z\right)\right)\right)\right)=z+\widetilde{v}$ and

$$
\begin{aligned}
& m\left(F_{2}, m\left(F_{1}, m\left(F_{3}, z\right)\right)\right) \\
& \quad=m\left(F_{1}, m\left(F_{1}, m\left(F_{2}, m\left(F_{1}, m\left(F_{3}, z\right)\right)\right)\right)\right) \\
& \quad=m\left(F_{1}, z+\widetilde{v}\right)=m\left(F_{1}, z\right)+\widetilde{M}\left(F_{1}\right) \widetilde{v} .
\end{aligned}
$$

According to these relationships, it can be established, for points satisfying $F_{3}^{T}\left(z_{p}-\eta_{\circ}\right)=1$,

$$
\begin{aligned}
\left|x-m\left(F_{1}, m\left(F_{2}, m\left(F_{1}, z\right)\right)\right)\right|_{P}^{2} & \geq\left|x-m\left(F_{3}, z\right)\right|_{P}^{2} \\
\left|x-m\left(F_{1}, m\left(F_{2}, m\left(F_{1}, m\left(F_{3}, z\right)\right)\right)\right)\right|_{P}^{2} & \geq|x-z|_{P}^{2}
\end{aligned}
$$

and

$$
\begin{aligned}
\left|x-m\left(F_{2}, m\left(F_{1}, z\right)\right)\right|_{P}^{2} & \geq\left|x-m\left(F_{1}, m\left(F_{3}, z\right)\right)\right|_{P}^{2} \\
\left|x-m\left(F_{2}, m\left(F_{1}, m\left(F_{3}, z\right)\right)\right)\right|_{P}^{2} & \left.\geq \mid x-m\left(F_{1}, z\right)\right)\left.\right|_{P} ^{2}
\end{aligned}
$$

These, and other relationships that follow by symmetry, establish that Assumption 3 holds.

\section{Simulation EXAmple}

Consider a rectangular billiard characterized by the vectors $F_{i}$ used in Section V-A:

$$
\left[\begin{array}{c|c|c|c}
F_{1} & F_{2} & F_{3} & F_{4}
\end{array}\right]=\frac{1}{5}\left[\begin{array}{c|c|c|c}
6 & -2 & -3 & 2 \\
2 & 6 & -1 & -6
\end{array}\right],
$$

and assume that $\alpha(z)=0$ and $\phi(x)=0$ in (2), so that $z$ and $x$ correspond to two masses moving on a flat surface.
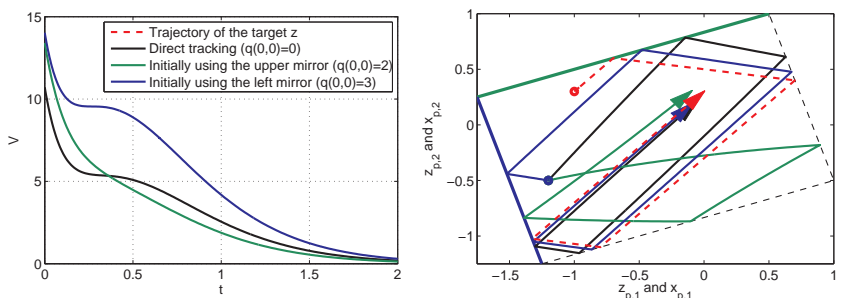

Fig. 1. Behavior of the Lyapunov function (left) and positions on the billiards for different initial conditions (right).

We satisfy the conditions in Section VI-A with $K=$ $\bar{K} \otimes I_{2 \times 2}$ where $\bar{K}=[-4-4]$ and $P=\bar{P} \otimes I_{2 \times 2}$ where $\bar{P}=\operatorname{diag}(4,1)$. According to Proposition 4 in Section VI-E we can satisfy Assumption 3 and use the control algorithm of Section V-B to achieve global tracking, according to Theorem 2. Figure 1 contains simulations of the closedloop response from $z(0,0)=\left[\begin{array}{llll}-1 & 0.3 & 1 & 1\end{array}\right]^{T}, x(0,0)=$

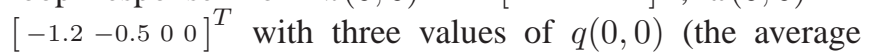
dwell-time variable $\sigma$ is not mentioned since it is not used in the control law). The $x$ mass starts with zero initial velocity. The $z$ mass is represented by the red-dashed trace in the right-hand figure and, as proved in Theorem 2, all three simulations achieve successful tracking. If $q(0,0)=0$ (black trace), the initial tracking error is $x-z$ and the controller forces the $x$ motion towards the north-east direction, where the $z$ mass is initially heading. If $q(0,0)=2$ (green trace), the upper mirror and the arising tracking error $x-n_{2}(z)$ are used at the initial time. Then, the controller forces the $x$ mass in the east direction because it compares it to the reflection of the $z$ ball given by the upper mirror. One way to interpret this is that the controller is initially "undo-ing" the first impact of the $z$ mass. Similarly, for $q(0,0)=3$ (blue trace), the left mirror is used at the initial time and the controller points towards the left wall. In this case, the controller is initially "undo-ing" the first impact of the $x$ mass.

\section{REFERENCES}

[1] B. Brogliato. Nonsmooth mechanics: models, dynamics, and control. Springer Verlag, 1999.

[2] C. Cai, A.R. Teel, and R. Goebel. Smooth Lyapunov functions for hybrid systems Part II:(pre) asymptotically stable compact sets. IEEE Transactions on Automatic Control, 53(3):734-748, 2008.

[3] F. Forni, A.R. Teel, and L. Zaccarian. Tracking control in billiards using mirrors without smoke, part I: Lyapunov-based local tracking in polyhedral regions. In Joint CDC-ECC, Orlando (FL), USA, December 2011, to appear.

[4] S. Galeani, L. Menini, A. Potini, and A. Tornambè. Trajectory tracking for a particle in elliptical billiards. Int. J. of Control, 81(2):189-213, February 2008.

[5] R. Goebel, R. Sanfelice, and A.R. Teel. Hybrid dynamical systems. Control Systems Magazine, IEEE, 29(2):28-93, April 2009.

[6] L. Menini and A. Tornambè. Asymptotic Tracking of Periodic Trajectories for a Simple Mechanical System Subject to Nonsmooth Impacts. IEEE Trans. Aut. Control, 46:1122-1126, 2001.

[7] R.G. Sanfelice, R. Goebel, and A.R. Teel. Invariance principles for hybrid systems with connections to detectability and asymptotic stability. IEEE Transactions on Automatic Control, 52(12):2282-2297, 2007.

[8] A.R. Teel, F. Forni, L. Zaccarian Lyapunov-based sufficient conditions for exponential stability in hybrid systems. IEEE transaction on Automatic Control, submitted. Also available at: http://control.disp.uniroma2.it/zack/ftp/submitted/ExpHybrid.pdf. 